\title{
Comparison of thermo-poroelastic and thermo- poroelastoplastic constitutive models to analyze THM process in clays
}

\author{
Mohammadreza Mir Tamizdoust ${ }^{1}$ and Omid Ghasemi-Fare ${ }^{1, *}$ \\ ${ }^{1}$ Department of Civil and Environmental Engineering, University of Louisville, USA
}

\begin{abstract}
Thermal pore pressurization in soil media has been investigated for the past few decades. It has been shown that temperature variations may significantly affect thermal pore pressure in clay soils confined deep into the ground. Moreover, thermal loading may lead to stress change and thermal deformation. Thermo-poroelastic and advance thermo-poroelastoplastic constitutive models have been formulated and incorporated numerically to simulate the thermo-hydromechanical process. However, the accurate response of soil media during THM process has not been completely understood. Although numerical modelling reasonably predicts the experimental observations, they still could not be used to completely justify the field observations. In this study, the main features of the thermo-poroelastic model are incorporated in a thermo-poroelastoplastic constitutive model (ACMEG-T) to further investigate the effect of different thermal and hydraulic properties on thermo-hydro-mechanical (THM) response of the soil media.
\end{abstract}

\section{Introduction}

Pore water pressure increases at elevated temperatures in normally and overconsolidated clays due to the difference of the thermal expansion coefficients of water and solid grains. Several research in literature studied the thermally-induced pore water pressure which is known as thermal pore pressurization through experimental and numerical modelling [1,2]. The thermal and hydraulic properties of water, and the porous media can greatly affect the thermal pore pressurization $[3,4]$. It has been shown that, thermal properties of the saturating water control the thermal pore pressurization and its diffusion process [5]. Moreover, according to the experimental observations, dissipation of pore water can be reversible or irreversible depending on the over consolidation ratio (OCR) [6].

The effects of thermal, hydraulic, and mechanical properties of the soil media on thermal pressurization have been considered through incorporating the poromechanics constitutive models. Thermallyinduced irreversible deformations were considered in these studies through developing temperaturedepended yielding criteria [7-9]. However, porous media and saturating water properties are highly temperature dependent (e.g., permeability of soil, viscosity, and volumetric expansion of water). Recently, Tamizdoust and Ghasemi-Fare [5] proposed a simple thermo-poroelastic model with precise consideration of temperature-dependent properties of the water to simulate the thermal pore pressurization in Boom clay. They found that a poro-elastic model can reasonably predict the thermal pressurization if changes in water properties with temperature are considered [5]. They also observed that even a small perturbation in porosity has major effect on pore water pressure variations.

In this study, a thermo-poroelastoplastic model is developed to investigate the influence of plastic deformation on thermal pore pressurization. First, the mathematical formulations are presented, and then will be incorporated in a coupled thermo-hydromechanical (THM) finite element model to simulate the thermal pore pressurization in Boom clay. The numerical results are compared with the field measurements at the underground research facility HADES-URF (ATLAS) [10].

\section{Mathematical model description}

In order to analyze the THM process in soil, mass balance equations of fluid and solid are coupled with soil stress equilibrium, and energy equation. All equations are formulated in Cartesian $(\mathrm{x}, \mathrm{y}, \mathrm{z})$ coordinate system, where: $\partial(\bullet) / \partial t, \nabla(\bullet), \nabla .(\bullet)$, and $\nabla^{2}(\bullet)$ are time derivate, gradient, divergence, and Laplace of field variables, respectively. In this study, the continuum balance and constitutive equations are described in the Eulerian configuration.

*Corresponding author: Omid.ghasemifare@1ouisville.edu 


\subsection{Macroscopic balance equations}

According to the mixture theory, the mass balance equations of the solid skeleton and fluid are as follows [11]:

$$
\begin{gathered}
\frac{\partial\left[(1-n) \rho_{s}\right]}{\partial t}+(1-n) \rho_{s} \nabla \cdot \mathbf{v}_{\mathbf{s}}=0 \\
\frac{\partial\left(n \rho_{f}\right)}{\partial t}+n \rho_{f} \nabla \cdot \mathbf{q}=0
\end{gathered}
$$

where $n\left(\mathrm{~m}^{3} / \mathrm{m}^{3}\right), \quad \rho_{f}\left(\mathrm{~kg} / \mathrm{m}^{3}\right)$, and $\rho_{s}\left(\mathrm{~kg} / \mathrm{m}^{3}\right)$ are the porosity, water density, and solid density, respectively. $\mathbf{v}_{\mathbf{s}}$ and $\mathbf{q}(\mathrm{m} / \mathrm{s})$ are the mass-averaged solid velocity and the fluid flux velocity, respectively. Equations (1) and (2) govern the mass balance in a two-phase medium where there is no mass exchange between the phases. The fluid flux velocity in soil is governed by Darcy's law:

$$
n \mathbf{q}=-\frac{k}{\mu}\left(\nabla p_{f}+g \nabla z\right)
$$

where $p_{f}(\mathrm{~Pa})$ is the pore fluid pressure, $k\left(\mathrm{~m}^{2}\right)$ is the intrinsic permeability of the medium, $\mu$ (Pa.s) is the dynamic viscosity of the fluid, and $g\left(\mathrm{~m} / \mathrm{s}^{2}\right)$ is the acceleration of gravity.

Solid particles are incompressible while the saturating water is slightly compressible. Soil stress equilibrium is also coupled with mass balance equation to predict the relation between the porosity and total stress variations. Soil stress equilibrium can be expressed as [11]:

$$
\nabla . \boldsymbol{\sigma}+\left[n \rho_{f}+(1-n) \rho_{s}\right] g=0
$$

where $\boldsymbol{\sigma}(\mathrm{Pa})$ is the total Cauchy stress which is decomposed to the Terzaghi's effective stress $\left(\boldsymbol{\sigma}^{\prime}\right)$ and pore fluid pressure $\left(p_{f}\right)$ as follows:

$$
\boldsymbol{\sigma}=\boldsymbol{\sigma}^{\prime}+p_{f} \mathbf{I}
$$

where $\mathbf{I}$ is the identity second-order tensor.

Effective stress is related to the deformation of the porous medium through a poromechanics constitutive model which will be expressed later.

In non-isothermal flow, energy balance equation governs the heat flow:

$$
\frac{\partial T}{\partial t}-\alpha_{m e} \nabla^{2} T=0
$$

where $\mathrm{T}\left({ }^{\circ} \mathrm{C}\right)$ is temperature. Subscript me denotes the medium, and $\alpha_{m e}\left(\mathrm{~m}^{2} / \mathrm{s}\right)$ is thermal diffusivity of the medium:

$$
\alpha_{m e}=\left(\frac{\lambda}{\rho C}\right)_{m e}=\frac{n \lambda_{f}+(1-n) \lambda_{s}}{n \rho_{f} C_{f}+(1-n) \rho_{s} C_{s}}
$$

$C_{f}$ and $C_{s}\left(\mathrm{~J} / \mathrm{kg} /{ }^{\circ} \mathrm{C}\right)$ are the specific heat capacities of the water and solid grains at constant stress, respectively. $\lambda_{f}$ and $\lambda_{s}\left(\mathrm{~W} / \mathrm{m} /{ }^{\circ} \mathrm{C}\right)$ are the thermal conductivities of the water and solid grains. In Equation (6), the convection and radiation of heat have been neglected due to very low permeability of clays. Therefore, it is expected heat conduction is the sole mode of heat transport in saturated clayey soil. Moreover, the solid and fluid phases are in thermal equilibrium ( $\left.T=T_{s}=T_{f}\right)$ because of the time associated heat transport between phases is very fast with respect to the other transport in the media.

Equations (2), (4), and (6) can govern the THM process in saturated soil media. However, the coupling thermal, hydraulic, and mechanical terms need to be elaborated. The coupling effects of temperature and stress on pore fluid pressure are through the changes in porosity and fluid density. Changes in soil porosity in the medium can be predicted using Equation (1) and considering the incompressibility of the solid grains:

$$
\frac{\partial n}{\partial t}=(1-n)\left[\frac{\partial \varepsilon_{\mathrm{vol}}}{\partial t}-\alpha_{s} \frac{\partial T}{\partial t}\right]
$$

where $\boldsymbol{\varepsilon}_{\mathrm{vol}}(\mathrm{m} / \mathrm{m})$ is the volumetric strain and can be composed of elastic (recoverable) and inelastic (irrecoverable) deformations, and $\alpha_{s}\left(1 /{ }^{\circ} \mathrm{C}\right)$ is the volumetric thermal expansion coefficient of solid grains. $\alpha_{s} \times(\partial T / \partial t)$ is the thermal strain of the solid particles and a negative sign represents the dilative deformation. Equation (8) indicates that the porosity change of the medium is governed by thermoporomechanics theory [5]. Variations of fluid density depends on thermal gradient and pore water pressure generation/ dissipation [12]:

$$
\frac{\partial \rho_{f}}{\partial t}=\rho_{f}\left[\left(\frac{1}{K_{f}}\right) \frac{\partial p_{f}}{\partial t}-\alpha_{f} \frac{\partial T}{\partial t}\right]
$$

where $K_{f}(1 / \mathrm{Pa})$ is the bulk modulus of water and $\alpha_{f}\left(1 /{ }^{\circ} \mathrm{C}\right)$ is the linear thermal expansion coefficient of the fluid. By substituting, Equations (8) and (9) in Equation (2) and considering the definition of the fluid flux velocity in Equation (3) we obtain the fluid mass conservation equation coupled with temperature and deformation field:

$$
\begin{gathered}
\frac{\partial p_{f}}{\partial t}-\frac{k}{\mu S} \nabla^{2} p_{f}=\Lambda \frac{\partial T}{\partial t}-\left(\frac{1-n}{S}\right) \frac{\partial \boldsymbol{\varepsilon}_{\mathrm{vol}}}{\partial t} \\
S=\frac{n}{K_{f}} ; \Lambda=\frac{n \alpha_{f}+(1-n) \alpha_{s}}{S}
\end{gathered}
$$

where $S(\mathrm{~Pa})$ is the storage term and $\Lambda\left({ }^{\circ} \mathrm{C} / \mathrm{Pa}\right)$ is the thermal pressurization coefficients, respectively. The effect of gravity is omitted in Equation (10a) since the horizontal flow is considered in this study. The thermal pressurization coefficients can be obtained experimentally through an undrained non-isothermal tests on saturated soils [13]. Equations (10a) and (10b) demonstrate that thermal pore pressurization happens because of the difference of the thermal expansion coefficients of solid and water. In addition, pressure diffusion depends on dynamic viscosity of water, and soil intrinsic permeability. As stated before, the 
volumetric strain in Equation (10a) depends on the constitutive model assumption (elastic, or elastoplastic). In this study, to predict the thermal plastic deformations, an advanced thermoporoelastoplastic model is adopted, and the results are compared with the previous model developed by the authors in which thermo-poroelastic model was considered.

\section{2. thermo-poroelastoplastic constitutive equation}

The thermo-poroelastoplastic model is based on bounding surface theory, which is comprised of isotropic and deviatoric yield surfaces, the same as ACMEG-T model [14] with a different choices of deviatoric yield surfaces (modified Cam-clay surface is used here). Table 1 presents formulations of the constitutive model used in this study. The model has 12 parameters which need to be identified.

Equations (T1) and (T2) define the thermoelastoplastic deformation which can affect the porosity in Equation (9). Here, the isotropic elastic behavior is assumed for Boom clay where the shear and bulk moduli are pressure dependent. According to the ACMEG-T constitutive model, the plastic evolution of isotropic (T3) and deviatoric (T4) yield surfaces are governed by $r_{i s o}$ and $r_{d e v}$, respectively. Moreover, the preconsolidation pressure is temperature dependent which controls the size of the yield surface at different temperatures (the yield surface shrinks at high temperature). The isotopic hardening rule is the same as the one defined in modified Cam-clay model. The slope of critical state line is also considered as temperature dependent and is incorporated in the model.

Furthermore, for complete description of coupled THM analysis, the temperature-dependency of fluid properties must be considered. An initial analysis is conducted to investigate the influence of variations of these properties with temperature (not shown here) on thermal pore pressurization, and the results indicate that the variations of $\alpha_{f}, \mu$, and $\lambda_{f}$ are significant and cannot be ignored. Therefore, the thermodynamic properties of water are calculated based on the mathematical relations provided by International Association for the Properties of Water and Steam (IAPWS) [15]:

$$
\begin{aligned}
& \alpha_{f}=\left(0.00005 T^{3}-0.01 T^{2}+2 T-6\right) \times 10^{-5} \\
& \mu=\left(-0.0002 T^{3}+0.05 T^{2}-4 T+178\right) \times 10^{-5} \\
& \lambda_{f}=-0.000007 T^{2}+0.0018 T+0.5711
\end{aligned}
$$

The range of validity of the Equations (11a)-(11c) is from 0 to $100{ }^{\circ} \mathrm{C}$.

\section{Numerical simulations}

\subsection{ATLAS experiment}

The theoretical model described above is utilized to simulate the THM process in Boom clay. Boom clay is a natural over consolidated deposit which has been considered as a suitable porous environment for deep geological repositories in Belgium [10]. Therefore, in order to validate the model, ATLAS experiment is selected which was conducted in HADES-URF underground research facility.

The ATLAS experiment consists of a horizontal main borehole (19 $\mathrm{m}$ long) with heaters and two parallel boreholes (15.65 $\mathrm{m}$ long) with instrumentations at the depth of $223 \mathrm{~m}$ in Boom clay. The observation boreholes for measurement have been drilled at $1.184 \mathrm{~m}$ and $1.515 \mathrm{~m}$ of the main borehole in the same horizontal plane. The changes in temperature, pore water pressure, and radial stress have been measured in both observation boreholes. The thermal loading associated with the ATLAS experiment during the first cooling phase is shown in Figure 1. Moreover, the physical properties of Boom clay and the material parameters used for the simulations are well documented in the literature and

\begin{tabular}{|c|c|c|c|}
\hline & No. & Equations & Description \\
\hline Elastic strain & $\mathrm{T} 1$ & $d \varepsilon^{\mathrm{e}}=\mathrm{d} \varepsilon-d \varepsilon^{\mathrm{p}}=C^{\mathrm{e}}: d \sigma^{\prime}$ & $\mathbf{C}^{\mathrm{e}}(\mathrm{Pa})$ : Compliance elasticity $4^{\text {th }}$ order tensor \\
\hline $\begin{array}{l}\text { Plastic strain } \\
\text { (flow rule) }\end{array}$ & $\mathrm{T} 2$ & $\mathbf{d} \varepsilon^{\mathbf{p}}=\sum_{i=1}^{2} \lambda_{i}^{p}\left(\partial Q_{i} / \partial \boldsymbol{\sigma}^{\prime}\right)$ & $\begin{array}{l}Q \quad(\mathrm{~Pa}) \text { : Plastic potential function } \\
\lambda^{p}(-) \text { : Plastic multiplier }\end{array}$ \\
\hline \multirow[b]{2}{*}{ Yield surface } & $\mathrm{T} 3$ & $f_{i s o}=p-p_{c T} r_{\text {iso }}$ & \multirow{2}{*}{$\begin{array}{l}p(\mathrm{~Pa}): \text { Effective mean stress } \\
p_{c T}(\mathrm{~Pa}) \text { : Non-isothermal preconsolidation pressure } \\
q(\mathrm{~Pa}): \text { Deviatoric stress } \\
r_{i s o} \text { and } r_{d e v}(-): \text { Isotropic and deviatoric degrees of } \\
\text { plastification, respectively } \\
M_{T}: \text { Non-isothermal slope of critical state line } \\
Q_{i}=f_{i} \text { and } i=i s o, \text { dev }: \text { Associate flow rule }\end{array}$} \\
\hline & $\mathrm{T} 4$ & $f_{d e v}=q^{2} / p+M_{T}^{2}\left(p-p_{c T}\right) r_{d e v}=0$ & \\
\hline
\end{tabular}
are presented in Table 2 [9].

Table 1. Formulation of thermo-poroelastoplastic constitutive model 
Table 2. THM properties of Boom clay.

\begin{tabular}{|c|c|c|c|}
\hline Parameters & Values & Parameters & Values \\
\hline$\rho_{f 0}\left(\mathrm{~kg} / \mathrm{m}^{3}\right)$ & 1000 & $v(-)$ & 0.125 \\
\hline$\rho_{s}\left(\mathrm{~kg} / \mathrm{m}^{3}\right)$ & 2670 & $E_{\text {ref }}(\mathrm{Pa})$ & $3.5 \times 10^{8}$ \\
\hline$n_{0}\left(\mathrm{~m}^{3} / \mathrm{m}^{3}\right)$ & 0.39 & $m(-)$ & 0.8 \\
\hline$k_{0}\left(\mathrm{~m}^{2}\right)$ & $2.5 \times 10^{-19}$ & $p_{\text {ref }}(\mathrm{Pa})$ & $2.48 \times 10^{6}$ \\
\hline$K_{f}(\mathrm{~Pa})$ & $2.17 \times 10^{9}$ & $\phi_{0}($ degree $)$ & 16 \\
\hline$C_{f}(\mathrm{~J} / \mathrm{kg} / \mathrm{K})$ & 4200 & $\gamma_{T}(-)$ & 0.55 \\
\hline$C_{s}(\mathrm{~J} / \mathrm{kg} / \mathrm{K})$ & 730 & $g_{T}(1 / \mathrm{K})$ & 0.0085 \\
\hline$\lambda_{s}(\mathrm{~W} / \mathrm{m} / \mathrm{K})$ & 1.65 & $r_{\text {iso }}^{e}(-)$ & 0.415 \\
\hline$\alpha_{s}(1 / \mathrm{K})$ & $1.3 \times 10^{-5}$ & $r_{\text {dev }}^{e}(-)$ & 0.3 \\
\hline$e_{0}\left(\mathrm{~m}^{3} / \mathrm{m}^{3}\right)$ & 0.639 & $a(-)$ & 0.014 \\
\hline$\beta(-)$ & 18 & $b(-)$ & 0.007 \\
\hline
\end{tabular}

Table 3. Initial in-situ condition.

\begin{tabular}{|c|c|}
\hline Parameters & Values \\
\hline$\sigma_{x}=\sigma_{y}=\sigma_{z}(\mathrm{~Pa})$ & $4.5 \times 106$ \\
\hline$p_{f 0}(\mathrm{~Pa})$ & $2.025 \times 106$ \\
\hline OCR & 2.4 \\
\hline$T_{0}\left({ }^{\circ} \mathrm{C}\right)$ & 16.5 \\
\hline
\end{tabular}

The governing balance equations and thermoporoelastoplastic constitutive model are simultaneously solved in COMSOL Multiphysics v5.3a. A $2 \mathrm{D}$ axisymmetric horizontal domain (xy plane) is considered where the heat source is placed at the symmetry boundary. A rectangular domain geometry with $100 \mathrm{~m}$ in perpendicular (x-direction) and $119 \mathrm{~m}$ in parallel (y-direction) direction to the heat source is accounted to minimize the effect of boundary conditions on the results (Figure 2). The insitu initial conditions of the experiment $(\mathrm{z}=223 \mathrm{~m}$ from the ground surface) are presented in Table 3 and are adopted in COMSOL.

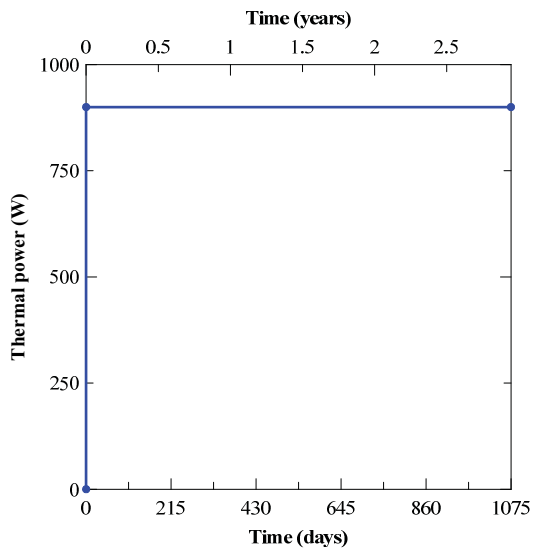

Fig. 1. Thermal power of the heater in ATLAS experiment.
Constant temperature and pore water pressure equal to the initial values (Table 3 ) are considered for all boundaries except the axisymmetric one. After series of mesh sensitivity analysis, the whole domain is discretized with 975 rectangular quadratic elements. Figure 2 presents the finite element meshes and a schematic view of the model. The set of PDEs presented in the previous section is implicitly solved using the PARADISO direct matrix solver which is available in the COMSOL Multiphysics software.

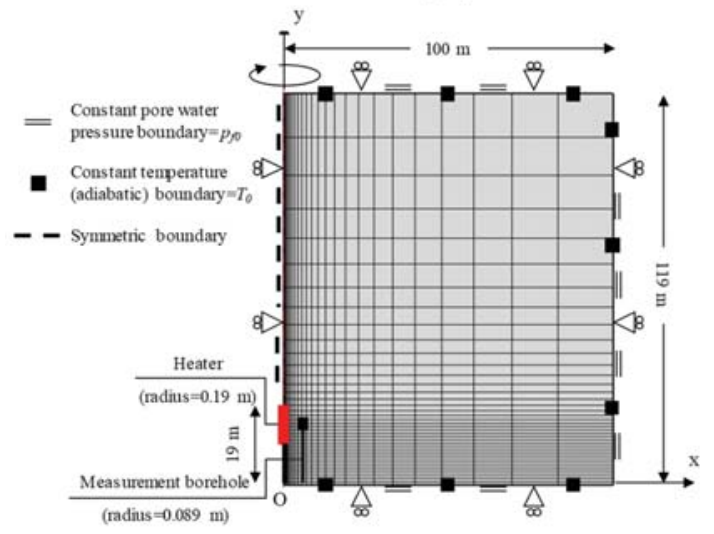

Fig. 2. Schematic representation of the finite element domain, initial, and boundary conditions.

\subsection{Results and discussion}

Evolution of temperature, thermal pore pressurization, changes in effective and total stress, and plastic deformations are obtained using the THM model. Figure 3 compares the numerical and experimental evolution of temperature at the instrumentation borehole $(\mathrm{x}=1.515 \mathrm{~m})$. The model simulates the temperature variations with decent accuracy. The thermo-poroelastic and thermoporoelastoplastic resulted the same temperature variation (only the result of the thermoporoelastoplastic approach is shown here). According to Equations (6) and (7), the porosity variations due to mechanical deformations can change the thermal diffusivity of the medium. However, variation of plastic porosity has minimal effect on thermal diffusivity, and therefore on temperature distribution.

Figures 4 (a) and (b) shows the simulated and measured thermal pore pressurization and orthoradial total stress $\left(\sigma_{y}\right)$ at $\mathrm{x}=1.515 \mathrm{~m}$ from the heater with respect to time. The results determine that considering variable properties of fluid with temperature leads to more accurate simulation. Figure 4(a) shows that thermo-poroelastoplastic model simulates thermal pressurization relatively better than thermo-elastic model with respect to the experimental observations. However, both approaches underestimate the experimental results. In Figure 4(b), thermoporoelastoplastic and thermo-poroelastic approaches yield somewhat the same results. The major 
difference between the simulation and experiment could not be capture by considering the effect of plastic deformation of thermal pressurization.

In addition, the effects of thermal expansion coefficient and dynamic viscosity of water are investigated and are presented in Figures 4 (a) and (b). In several THM models in the literature, these properties are considered as constant through the analysis [16]; however, as it can be seen in the figures, the impact of $\alpha_{f}$ and $\mu$ on thermal pore pressurization and stress field is paramount and cannot be ignored. According to Equation 10 (a) and (b), the thermal expansion coefficient directly affects the magnitude of thermal pore pressurization while the dynamic viscosity is one of the key factors that controls the excessive pore pressure dissipation. By comparing different results in Figures4 (a) and (b), it can be realized that although temperature dependency of thermal and hydraulic properties of the fluid may not have high impact on thermal pore pressurization for the presented heating load; However, during higher temperature these effects might be significant. Figure 5 presents thermo-plastic deformations at different radial distances from the heater. It shows that the plastic deformation initiates from the beginning of the heating and reaches to its maximum value at the end of the second heating phase.

It is illustrated in Figure 5 that plastic deformation is evident at least for $1.6 \mathrm{~m}$ away from the heat source. The influential zone for plastic deformation may become larger upon higher thermal loadings. Moreover, irreversible plastic strains may alter the mechanical and hydraulic characteristics of soils upon cooling phase, e.g. thermal consolidation which leads to permeability reduction. These effects might be important depending on the overconsolidation ratio and activity of clays and cannot be ignored.

Figure 6 displays that the radial influence zone of thermal loading on porosity is about $15 \mathrm{~m}$ from the heater. However, the porosity variations are

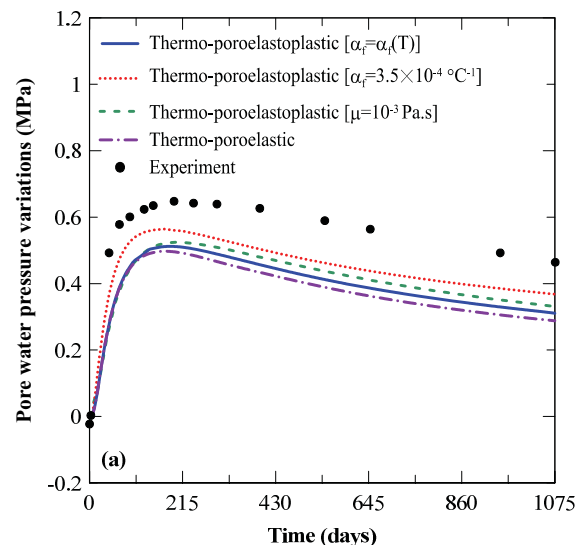

irreversible only for the zone close to the heater $(x<5$ $\mathrm{m})$. Moreover, the figures shows the difference in porosity variations obtained by thermoporoelastoplastic compared to thermo-poroelastic model where the plastic deformation facilitates the porosity reduction during heating and acts in oppose to the expansive thermal elastic deformations which are increasing the porosity.

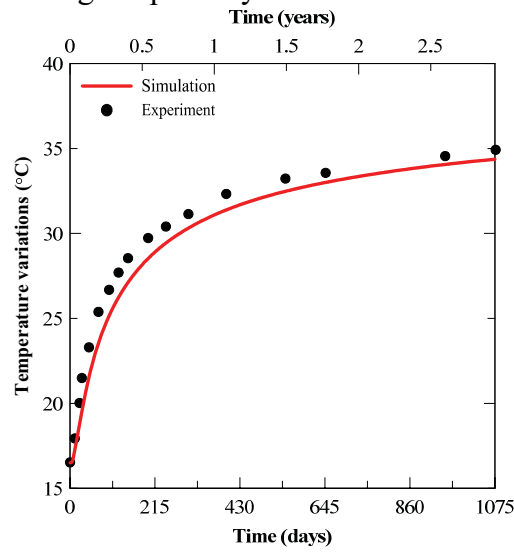

Fig. 3. Variations of temperature at $x=1.515 \mathrm{~m}$ from the heat source.

\section{Conclusion}

In this study, a numerical THM analysis is adopted to investigate the THM process in Boom clay. Thermoporoelastic and thermo-poroelastoplastic approaches with careful consideration of temperature-dependent water properties are utilized to compare the impact of different thermal, hydraulic, and mechanical properties of the medium. It is found that in the heating phase considered in this study plastic deformation improves the prediction of thermal pressurization. However, no significant effect on the temperature variations is observed.

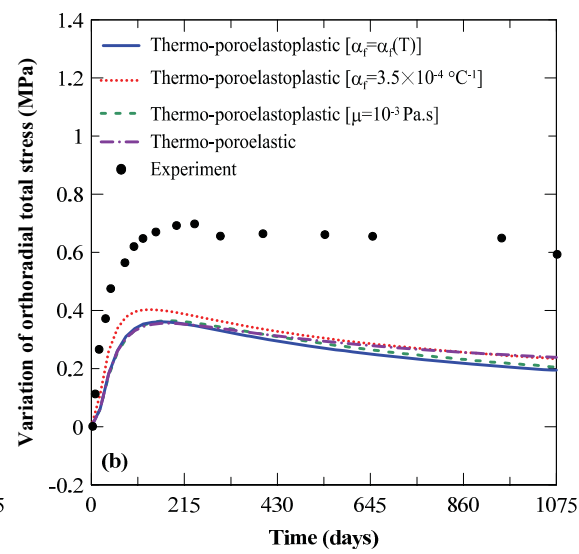

Fig. 4. Comparison of simulation results of thermo-poroelastoplastic (variable and constant $\alpha_{f}$ and $\mu$ ) with thermoporoelastic (variable $\alpha_{f}$ and $\mu$ ): (a) Evolution of thermal pore pressurization, and (b) orthoradial total stress at $\mathrm{x}=1.515 \mathrm{~m}$ from the heat source. 
The temperature dependency of thermal expansion coefficient and dynamic viscosity of water did not influence the production of thermal pressurization for the presented thermal loading; however, this might not be the case for high level of temperatures (about $\left.100{ }^{\circ} \mathrm{C}\right)$.

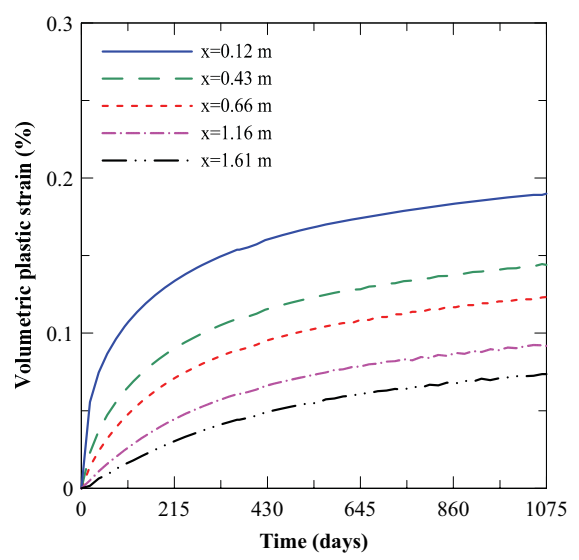

Fig. 5. Volumetric plastic strain at different distances from the heater.

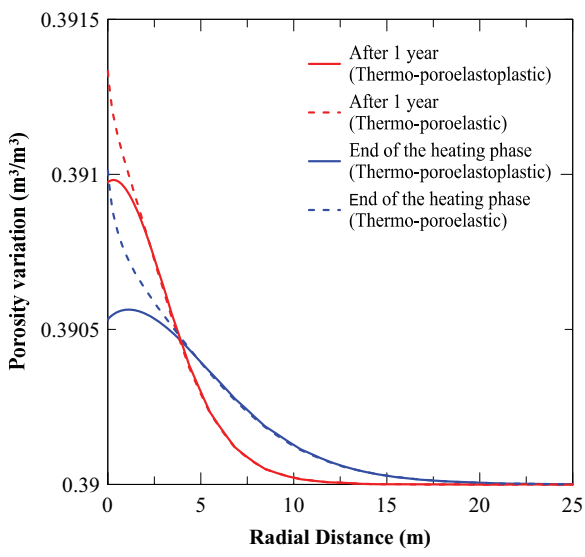

Fig. 6. Porosity change of Boom clay along the radial distance from the heater at different times.

The authors gratefully acknowledge the financial support by the National Science Foundation under Grant No. CMMI1804822 .

\section{References}

[1] Ghabezloo S, Sulem J. Stress dependent thermal pressurization of a fluid-saturated rock. Rock Mechanics and Rock Engineering. 2009;42(1):1.

[2] Joshaghani M, Ghasemi-Fare O, Ghavami M. Experimental Investigation on the effects of temperature on physical properties of sandy soils. IFCEE 20182018. p. 675-85.

[3] Braun P, Ghabezloo S, Delage P, Sulem J, Conil $\mathrm{N}$. Theoretical analysis of pore pressure diffusion in some basic rock mechanics experiments. Rock
Mechanics and Rock Engineering. 2018;51(5):136178.

[4] Tamizdoust MM, Ghasemi-Fare O. Coupled Thermo-Hydro-Mechanical Modeling of Saturated Boom Clay. Geo-Congress 2020: Geo-Systems, Sustainability, Geoenvironmental Engineering, and Unsaturated Soil Mechanics: American Society of Civil Engineers Reston, VA, 2020. p. 340-8.

[5] Tamizdoust MM, Ghasemi-Fare O. A Fully Coupled Thermo-Poro-Mechanical Finite Element Analysis to predict the Thermal Pressurization and Thermally Induced Pore Fluid Flow in Soil Media. Computers and Geotechnics. 2019, 117, 103250.

[6] Abuel-Naga HM, Bergado DT, Bouazza A. Thermally induced volume change and excess pore water pressure of soft Bangkok clay. Engineering Geology. 2007;89(1-2):144-54.

[7] Hueckel T, Borsetto MJJoge. Thermoplasticity of saturated soils and shales: constitutive equations. 1990;116(12):1765-77.

[8] Cui YJ, Sultan N, Delage P. A thermomechanical model for saturated clays. Canadian Geotechnical Journal. 2000;37(3):607-20.

[9] François B, Laloui L, Laurent C. Thermo-hydromechanical simulation of ATLAS in situ large scale test in Boom Clay. Computers and Geotechnics. 2009;36(4):626-40.

[10] De Bruyn D, Labat S. The second phase of ATLAS: the continuation of a running THM test in the HADES underground research facility at Mol. Engineering Geology. 2002;64(2-3):309-16.

[11] Coussy O. Poromechanics: John Wiley \& Sons, 2004.

[12] Delaney PT. Rapid intrusion of magma into wet rock: groundwater flow due to pore pressure increases. Journal of Geophysical Research: Solid Earth. 1982;87(B9):7739-56.

[13] Mohajerani M, Delage P, Sulem J, Monfared M, Tang AM, Gatmiri B. A laboratory investigation of thermally induced pore pressures in the CallovoOxfordian claystone. International Journal of Rock Mechanics and Mining Sciences. 2012;52(112-21.

[14] Laloui L, François B. ACMEG-T: soil thermoplasticity model. Journal of engineering mechanics. 2009;135(9):932-44.

[15] Spang B. Excel add-in for properties of water and steam in SI-units. Water97_v13 xla Hamburg. 2002. [16] Chen W, Ma Y, Yu H, Li F, Li X, Sillen X. Effects of temperature and thermally-induced microstructure change on hydraulic conductivity of Boom Clay. Journal of Rock Mechanics and Geotechnical Engineering. 2017;9(3):383-95. 